\title{
WINDOWED LEAST-SQUARES SPECTRAL ANALYSIS OF GRACE K-BAND RANGE RATE MEASUREMENTS
}

\author{
MORADI, A. ${ }^{*}$ - SHARIFI, M. A. \\ School of Surveying and Geomatics Engineering, College of Engineering, University of Tehran \\ North Kargar Ave., P.O. Box 11365-4563, Tehran, Iran \\ (phone: +98-918-162-2986; fax: +98-86-33670020) \\ ${ }^{*}$ Corresponding author \\ e-mail:amoradi90@ut.ac.ir \\ (Received 12 $2^{\text {th }}$ Sep 2016; accepted $1^{\text {st }}$ Dec 2016)
}

\begin{abstract}
The objective of this manuscript is to utilize windowed least-squares spectral analysis for extracting the frequency contents of GRACE K-band range rate time series related to Iran's main catchments. The spectral behavior of the unequally spaced time series affected by satellites and Earth rotations should be studied using the Least-Squares Spectral Analysis due to the inherent limitations in the traditional Fourier and Wavelet Transformations techniques. We explain the principles of windowed least- squares spectral analysis as an alternative method of the Least-Squares Spectral Analysis in order to access a time-frequency representation of irregularly sampled GRACE range rate time series related to Iran's main catchments. The results are in good agreement with the spectral behavior of the total water storage changes modeled in the catchments, as well as with the previous research findings.
\end{abstract}

Keywords: Iran's main catchments; hydrology; twin-satellite mission; least squares approximation; time series analysis

\section{Introduction}

The classical Least-Squares Spectral Analysis (LSSA) method was first introduced by Vanicek (1969) as an alternative to Fourier analysis, the most commonly used spectral approach in science. This analysis was developed in order to overcome some of the limitations of the Fourier technique; most importantly the requirement that the data is equally spaced and equally weighted with no gaps and datum shifts (Hui and Pagiatakis, 2004).

Both Fourier and classical LSSA use trigonometric functions in different frequencies as the basis for analyzing signals. This choice provides a very good frequency resolution and determines the frequency contents of the signal without any information about the corresponding time of occurrence of these frequencies. Currently, the most popular and useful method of time- frequency analysis is the wavelet transformation in which the base functions will be the waveforms of limited duration, instead of sinusoids extending from minus to plus infinity. Similar to Fourier analysis, wavelet transformation also requires equally spaced and equally weighted data. Fortunately, this shortcoming has been addressed in the second generation wavelets, which are capable of applying the unevenly data without any spectral interpretation (Sweldens, 1998). In this paper, by formulating a windowed transformation scheme, the weighted leastsquares approximation is introduced. The experimental studies assessing this method are conducted using the time series obtained from GRACE level 1B observations related to Iran's main catchments with the related gaps and weights.

The Gravity Recovery and Climate Experiment (GRACE) mission, launched in March 2002, has globally mapped the temporal variations of the Earth's gravity. The 
spatio-temporal alteration of the precisely measured distance between two satellites is affected by the mass changes in the Earth system (Han et al., 2005). Many studies have used different types of GRACE data, individually or together with other remote sensing or land surface observations, in order to monitor water storage pattern globally and regionally (see for example, Fatolazadeh et al., 2015; Lemoine et al., 2007; Rowlands et al., 2010; Tourian et al., 2015 and Voss et al., 2013).

In all of the above mentioned approaches, the desired hydrological signals extracted from GRACE measurements have been converted to different formats such as spherical harmonic coefficients and equivalent water thicknesses of juxtaposed tiles, resulting in missing information during the smoothing process. Since all of the mentioned products have been obtained from the GRACE K-band range rate measurements, we decided to use them directly in the present research.

In this manuscript we first present the mathematical foundations of Windowed Least-Squares Spectral Analysis (WLSSA). Then, we discuss its application for extracting the spectral behavior of the range rates time series related to Iran's main catchments. Lastly, we compare the results of the analysis of the time series with those of the monthly modeled Total Water Storage (TWS) changes related to the catchments extracted from WaterGAP (Water - a Global Assessment and Prognosis) hydrological model (Döll et al., 2003).

\section{Mathematical foundations}

The proposed method, as an application of Least-Squares Approximation (LSA) (Vaníček and Wells, 1972) is similar to LSSA, which is connected to the linear least squares parametric adjustment (Wells et al., 1985).

Given a vector of observations $f$, sampled at uniform or non-uniform time instants, we can set up a parametric model as follows:

$$
f(t)=\sum_{i} c_{i} \varphi_{i}(t)=\Phi * c
$$

Where, $\Phi$ is a matrix consisting of several column vectors, as base functions. Each function is of the same dimension as $\boldsymbol{f}$, and $\boldsymbol{c}$ is the vector of unknown coefficients.

In the classical LSSA, the form of the base functions is selected to be sines and cosines of different frequencies $\omega_{j}, j=1,2, \ldots, m$, and for each frequency, the best fitting approximant $\boldsymbol{p}$ to $\boldsymbol{f}$ will be obtained by minimizing the residual vector $\widehat{\boldsymbol{v}}=\boldsymbol{f}-\boldsymbol{p}$ in the least-squares sense as:

$$
\hat{\mathbf{c}}=\left[\hat{c}_{1} \hat{c}_{2}\right]^{T}=\left(\Phi^{T} \Phi\right)^{-1}\left(\Phi^{T} \boldsymbol{f}\right) \Rightarrow \boldsymbol{p}\left(\omega_{j}\right)=\hat{c}_{1} \cos \omega_{j} t+\hat{c}_{2} \sin \omega_{j} t
$$

Then the fractional content $s\left(\omega_{j}\right)$ of $\boldsymbol{f}$ represented by $\boldsymbol{p}\left(\omega_{j}\right)$ can be measured by:

$$
s\left(\omega_{j}\right)=\frac{f^{\mathrm{T}} p\left(\omega_{j}\right)}{f^{T} f}
$$

In the following subsection, the windowed least-squares spectral analysis is developed by the use of truncated or weighted sines and cosines as the base functions in the parametric model represented by Eq.1. 


\section{Windowed least-squares spectral analysis}

In order to estimate the frequency content of local sections of a signal sampled at uniform or non-uniform instants, the sinusoidal base functions can be multiplied by a window function, which is nonzero for only a short period of time, exactly similar to the Short-Time Fourier Transform (STFT) (Okamura, 2011). Therefore, the parametric model (Eq.1) can be rewritten as:

$$
\forall j ; \boldsymbol{f}=\left[\begin{array}{c}
f_{1} \\
\vdots \\
f_{n}
\end{array}\right]=\left[\begin{array}{cc}
\mathrm{w}\left(t_{1}-\tau\right) \cos \omega_{j} t_{1} & \mathrm{w}\left(t_{1}-\tau\right) \sin \omega_{j} t_{1} \\
\vdots & \\
\mathrm{w}\left(t_{n}-\tau\right) \cos \omega_{j} t_{n} & \mathrm{w}\left(t_{n}-\tau\right) \sin \omega_{j} t_{n}
\end{array}\right]\left[\begin{array}{c}
c_{1} \\
c_{2}
\end{array}\right]=\boldsymbol{\Phi} * \boldsymbol{c} \text { (Eq.4) }
$$

With the Gaussian window function:

$$
w(t)=\sqrt{\alpha / \pi} e^{-\alpha t^{2}}
$$

Where, $\alpha$ determines the length of the window. The least-squares estimation of the coefficients $c_{1}$ and $c_{2}$ and consequently, the best fitting approximant $\boldsymbol{p}\left(\tau, \omega_{j}\right)$ to $\boldsymbol{f}$ for each selected frequency $\omega_{J}$ and translation parameter $\tau$ will be computed as follows:

$$
\left[\begin{array}{l}
\hat{c}_{1} \\
\hat{c}_{2}
\end{array}\right]=\left(\Phi^{T} \Phi\right)^{-1}\left(\Phi^{T} f\right) \Rightarrow \boldsymbol{p}\left(\tau, \omega_{J}\right)=\hat{c}_{1} \mathrm{w}(\mathrm{t}-\tau) \cos \omega_{J} t+\hat{c}_{2} \mathrm{w}(\mathrm{t}-\tau) \sin \omega_{J} t \text { (Eq.6) }
$$

Finally, the spectral value corresponding to the selected frequency and translation parameter is obtained as:

$$
s\left(\tau, \omega_{J}\right)=\frac{f^{T} p\left(\tau_{z} \omega_{J}\right)}{f^{\mathrm{T}} f}
$$

Unlike the classical LSSA (Eq.3), spectrum calculated by this method (Eq.7) not only contains the information about the frequency contents of the signal but also the time of occurrence of these frequencies.

\section{Multivariate analysis}

The proposed WLSSA can be used to detect common components in multivariate time series with the ability to simultaneously focus on the time and frequency behavior of the signals. If several (r) time series with identical design matrix $\Phi$ exist in a linear model, the model will be referred to a multivariate linear model (Amiri-Simkooei and Asgari, 2011). In this case, the generalized form of the parametric model (Eq.1) will be:

$$
\operatorname{vec}(\boldsymbol{f})=\left(I_{r} \otimes \Phi\right) * \operatorname{vec}(\boldsymbol{c})
$$

Where, vec is the vector operator and $\otimes$ is the Kronecker product. If the series are uncorrelated with covariance matrix $\Sigma=\operatorname{diag}\left(\sigma_{11}, \sigma_{22}, \ldots, \sigma_{\mathrm{rr}}\right)$, the power spectrum is:

$$
s\left(\tau, \omega_{j}\right)=\sum_{i=1}^{r} s_{i}\left(\tau, \omega_{j}\right) / \sigma_{i i}
$$


That is, the weighted sum of the individual spectral values.

\section{Time series creation and analysis}

Presence of numerous processing methods for GRACE data has led to different types of released data products at several levels (Chen, 2007). In this study, the inter-satellite K-band range rate (KBRR) measurements with an accuracy of $0.1 \mu \mathrm{m} / \mathrm{s}$ and 5 seconds sampling, included in the products labeled L1B, in the period of January 2003 to December 2011 are used (GRACE LEVEL 1B JPL RELEASE 2.0. Ver. 2. PO.DAAC, CA, USA) (Case et al., 2002). Since the spatio-temporal variation of the GRACE Kband range rate measurements shows the mass changes at the surface of and within the Earth, the range rate time series corresponding to a selected area contains regional information about temporal variations of the gravity field, caused by fluctuations in total water storage, provided that the contributions to range rates from tide and nongravitational accelerations measured by the GRACE onboard accelerometers are reduced. In this study, the tides are modeled as variation to the spherical harmonic coefficients according to IERS Conventions, 2010 (Petit and B. Luzum, 2010), then the simulated perturbed orbits of the two GRACE satellites are generated by adding these variations to the static gravity field of the Earth, EGM (Earth Gravitational Model) 2008, and the tidal corrections are estimated by comparing the synthesized range rates derived from perturbed and unperturbed orbits. In the case of non-gravitational accelerations, a similar procedure is used, with the difference that these accelerations have to be calibrated before use in the perturbed orbit simulation. We estimate the accelerometer parameters by comparison with Precise Orbit Determination (POD) based non gravitational accelerations; for details, see Bezděk (2010).

As a case study, in the present paper, the above mentioned time series are produced by monthly averaging of the reduced observations related to the Iran's main catchments: Caspian Sea, Oromieh (Urmia) Lake, Serakhs (Ghareghoum), Central, Hamoon and Persian gulf (Fig. 1).

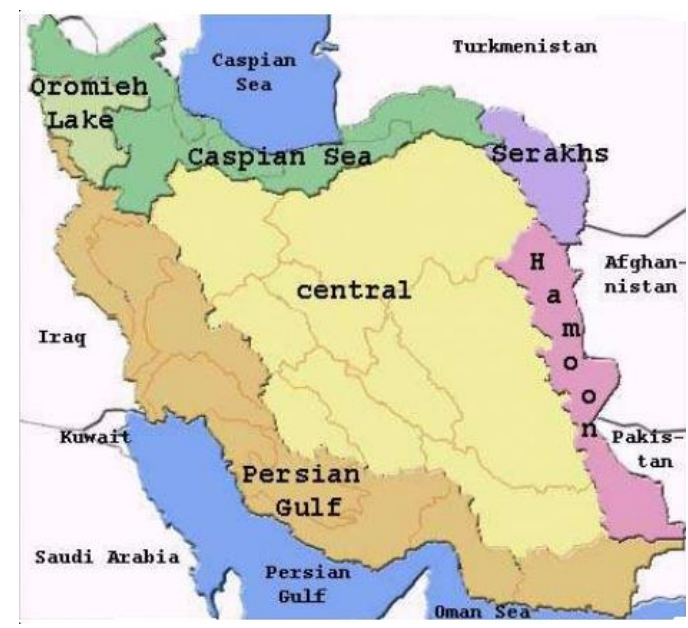

Figure 1. Map of the main catchments of Iran.

Based on the degree of relevance of each observation to a catchment, a weight that is proportional to the percentage of the catchment area covered by the instantaneous 
relative position vector between two satellites is assigned to that observation and the monthly quantities are computed as a weighted mean. The estimated monthly range rates are carrying various weights because of the different observational weights and also the difference in numbers of measurements therein a catchment in a month. At the same time, there are some months without any observed quantity in some catchments. This leads to unevenly spaced time series in these cases which cannot be analyzed using Fourier transformation or STFT. The extracted range rates are shown in Fig. 2.

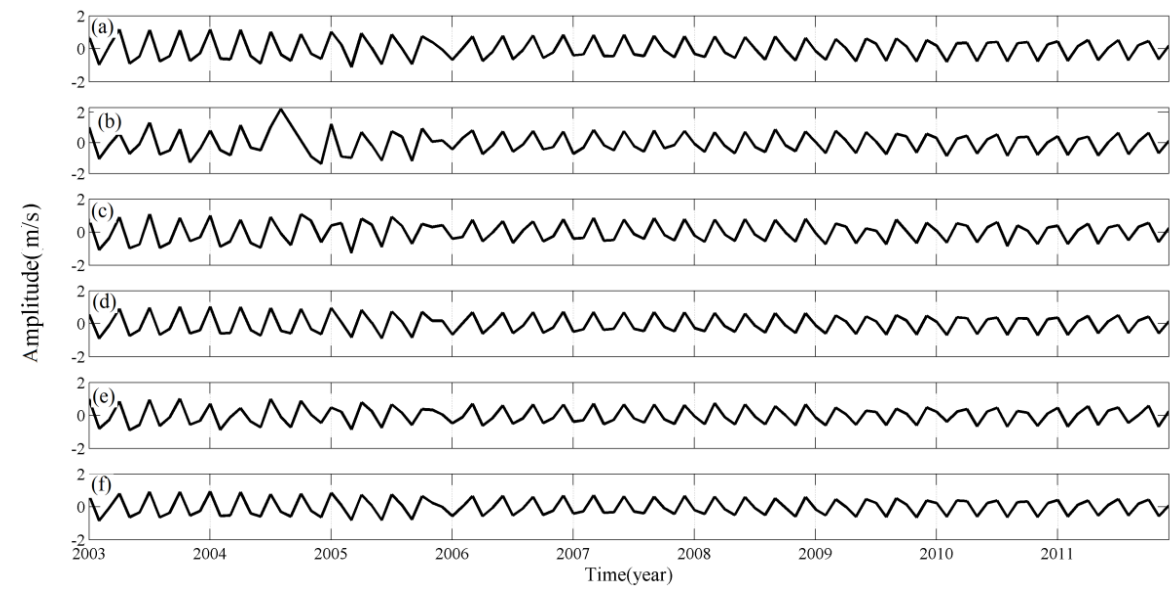

Figure 2. The range rate time series related to the main catchments of Iran: (a) Caspian Sea,

(b) Oromieh Lake, (c) Serakhs, (d) Central, (e) Hamoon and (f) Persian gulf.

After removing the previously known periods in the range rate signals associated with the GRACE orbital configuration (Visser, 2005), the classical LSSA and the proposed WLSSA are applied to the filtered time series to extract the time-frequency contents affected by the regional hydrology and the results are depicted in Fig 3.
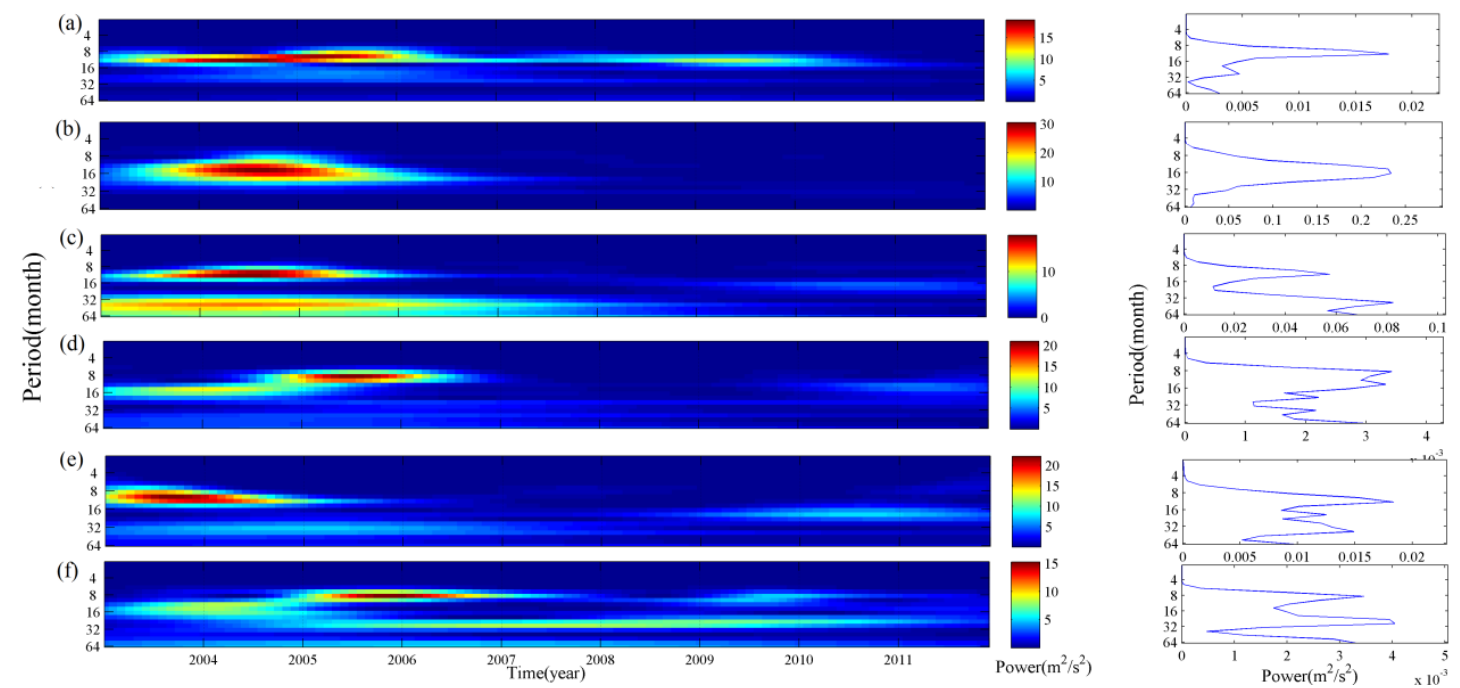

Figure 3. The estimated periodograms of the range rate time series related to the main catchments of Iran: (a) Caspian Sea, (b) Oromieh Lake, (c) Serakhs, (d) Central, (e) Hamoon and (f) Persian gulf, as the results of applying the classical LSSA (right) and WLSSA (left). 
As it can be seen in the above periodograms, a main constituent with the period of about 12 months exists in all signals corresponding to the main hydrological cycle in the catchments rooted in the annual circulation of the atmosphere.

The considerable expected time-frequency analyzing of the time series, as the specific result of using the WLSSA instead of the classical LSSA, implies that the annual constituent is not stationary. This explains the intensity of some of the spectral values in each shown periodogram. The identifiable decreases in the spectral values can be explained as the effect of the reported drought in the region containing Iran started around 2007 and 2008 (Joodaki et al., 2014; Voss et al., 2013). This result has been reported for Urmia catchment in Tourian et al. (2015), too.

In order to validate the results of the analysis, the monthly total water storage changes between 2003 and 2011 related to each catchment were obtained from WaterGAP hydrological model (Fig. 4) and were analyzed using the two abovementioned methods (Fig. 5).

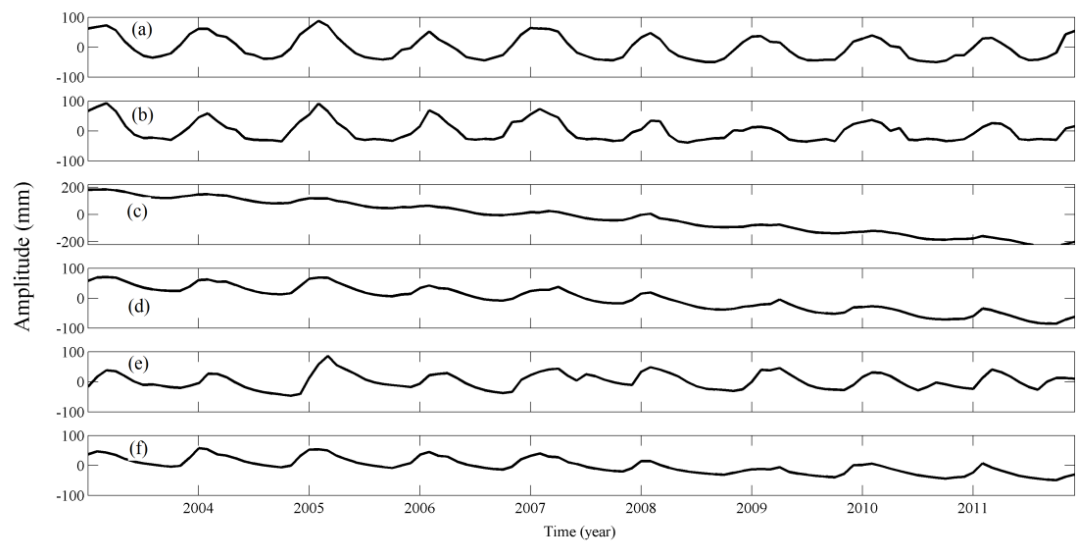

Figure 4. The modeled TWS changes related to the main catchments of Iran: (a) Caspian Sea, (b) Oromieh Lake, (c) Serakhs, (d) Central, (e) Hamoon and (f) Persian gulf.
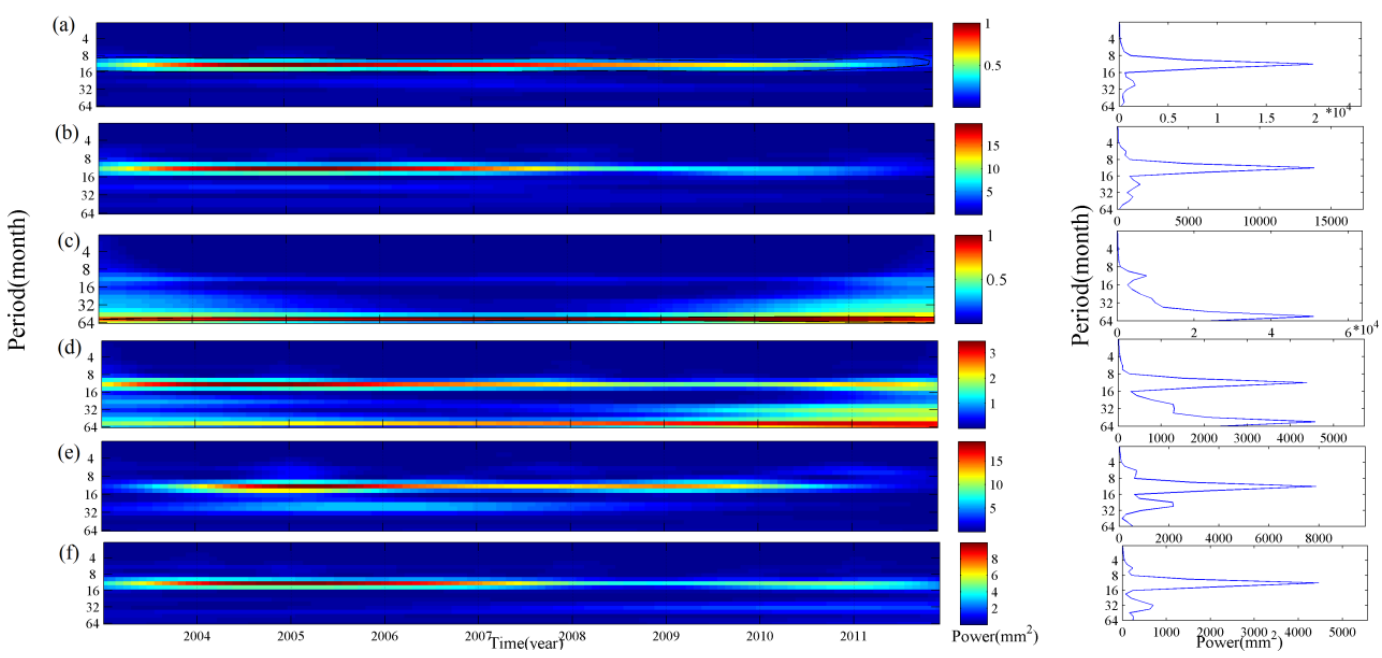

Figure 5. The estimated periodograms of the modeled TWS changes related to the main catchments of Iran: (a) Caspian Sea, (b) Oromieh Lake, (c) Serakhs, (d) Central, (e) Hamoon and (f) Persian gulf, as the results of applying the classical LSSA (right) and WLSSA (left). 
We expected that the one-year alternation occur in each TWS change signal again. However, we noted that all signals have been weakened after 2007 in all catchment areas except Serakhs catchment, in which the annual spectral contents started to decrease with a 2-year delay. This difference can also be seen in a similar fashion in the corresponding periodogram for the range rates.

In addition to the individual analysis, the multivariate analyzing of the both range rates and TWS change time series using the LSSA and WLSSA resulted in the multivariate periodograms shown in Fig. 6. The results are almost similar to those of individual signals, implying the weakness of annual constituent after 2007, as the main common component of the time series.
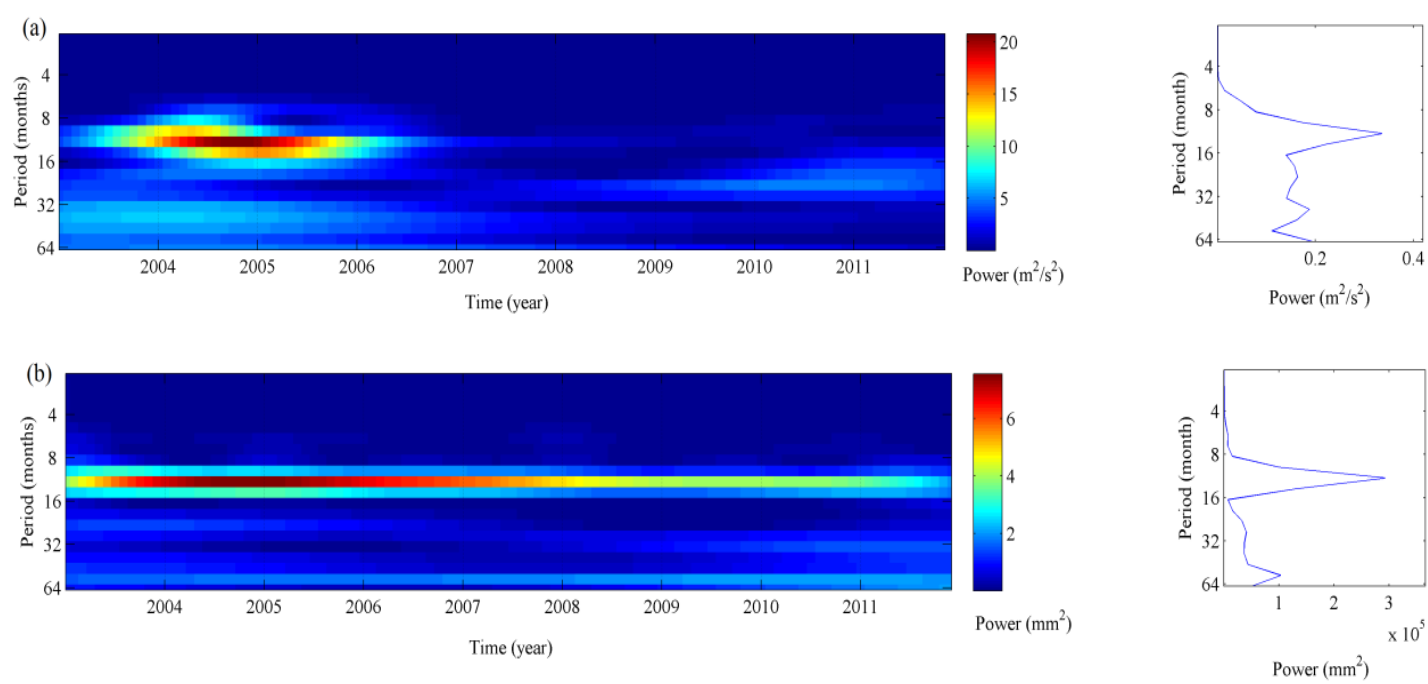

Figure 6. The estimated multivariate periodograms of $(a)$ the range rates and $(b)$ the modeled TWS changes related to the main catchments of Iran, as the results of applying the classical LSSA (right) and WLSSA (left).

\section{Conclusions}

In this study, the windowed least-squares spectral analysis was discussed as an alternative method to the classical LSSA for studying the time-frequency behavior of GRACE K-band range rate time series.

As a case study, the monthly averaged range rates related to the main catchments of Iran were analyzed. Each catchment time series had a main element with the period of about 12 months. This annual component is affected by the total water storage variations in the region and the significant decrease of its corresponding spectral values is most likely the result of the desiccation that started after 2007.

Monthly modeled TWS changes over each selected catchment were analyzed using the same analyzing tools. This led to extracting the spectral behavior of the annual component which has weakened after 2007.

Multivariate analyzing of both monthly range rates and TWS changes confirms the above mentioned time-frequency behavior as common contents of the signals related to the selected catchments.

The results of GRACE level 1B measurements related to the regions under study are in good agreement with similar information sources and those of previous researches. 
This indicates that these measurements could directly be used as an alternative to the conventional schemes.

Acknowledgements. The authors would like to thank Hannes Müller Schmied from Goethe University Frankfurt for providing WaterGAP TWS data from the newest model version.

\section{REFERENCES}

[1] Amiri-Simkooei, A.R., Asgari, J. (2012): Harmonic analysis of total electron contents time series: methodology and results. - GPS solutions 16(1): 77-88.

[2] Bezděk, A. (2010): Calibration of accelerometers aboard GRACE satellites by comparison with POD-based nongravitational accelerations. - Journal of Geodynamics 50(5): 410-423.

[3] Case, K., Kruizinga, G., Wu, S. (2002): GRACE level 1B data product user handbook. - JPL Publication D-22027.

[4] Chen, Y. (2007): Recovery of terrestrial water storage change from low-low satellite-tosatellite tracking. - Doctoral dissertation, The Ohio State University.

[5] Döll, P., Kaspar, F., Lehner, B. (2003): A global hydrological model for deriving water availability indicators: model tuning and validation. - Journal of Hydrology 270(1): 105134.

[6] Fatolazadeh, F., Voosoghi, B., Naeeni, M.R. (2016): Wavelet and Gaussian Approaches for Estimation of Groundwater Variations Using GRACE Data. - Groundwater 54(1): 7481.

[7] Han, S.C., Shum, C.K., Jekeli, C., Alsdorf, D. (2005): Improved estimation of terrestrial water storage changes from GRACE. - Geophysical Research Letters 32(7).

[8] Hui, Y., Pagiatakis, S. (2004): Least squares spectral analysis and its application to superconducting gravimeter data analysis. - Geo-Spatial Information Science 7(4): 279283.

[9] Joodaki, G., Wahr, J., Swenson, S. (2014): Estimating the human contribution to groundwater depletion in the Middle East, from GRACE data, land surface models, and well observations. - Water Resources Research 50(3): 2679-2692.

[10] Lemoine, J.M., Bruinsma, S., Loyer, S., Biancale, R., Marty, J.C., Perosanz, F., Balmino, G. (2007): Temporal gravity field models inferred from GRACE data. - Advances in Space Research 39(10): 1620-1629.

[11] Okamura, S. (2011): The short time Fourier transform and local signals. - Doctoral dissertation, Carnegie Mellon University.

[12] Petit, G., Luzum, B. (2010): IERS conventions 2010. - International earth rotation and reference systems service (No. 36). IERS Technical Note.

[13] Rowlands, D.D., Luthcke, S.B., McCarthy, J.J., Klosko, S.M., Chinn, D.S., Lemoine, F.G., Boy, J.P., Sabaka, T.J. (2010): Global mass flux solutions from GRACE: a comparison of parameter estimation strategies-mass concentrations versus Stokes coefficients. - Journal of Geophysical Research: Solid Earth 115(B1).

[14] Sweldens, W. (1998): The lifting scheme: A construction of second generation wavelets. - SIAM Journal on Mathematical Analysis 29(2): 511-546.

[15] Tourian, M.J., Elmi, O., Chen, Q., Devaraju, B., Roohi, S., Sneeuw, N. (2015): A spaceborne multisensor approach to monitor the desiccation of Lake Urmia in Iran. Remote Sensing of Environment 156: 349-360.

[16] Vaníček, P. (1969): Approximate spectral analysis by least-squares fit. - Astrophysics and Space Science 4(4): 387-391.

[17] Vaníček, P., Wells, D.E. (1972): The least squares approximation and related topics. - Department of Surveying Engineering, University of New Brunswick. 
[18] Visser, P.N.A.M. (2005): Low-low satellite-to-satellite tracking: a comparison between analytical linear orbit perturbation theory and numerical integration. - Journal of Geodesy 79(1-3): 160-166.

[19] Voss, K.A., Famiglietti, J.S., Lo, M., Linage, C., Rodell, M., Swenson, S.C. (2013): Groundwater depletion in the Middle East from GRACE with implications for transboundary water management in the Tigris-Euphrates-Western Iran region. - Water resources research 49(2): 904-914.

[20] Wells, D.E., Vaníček, P., Pagiatakis, S.D. (1985): Least squares spectral analysis revisited. - Fredericton, Canada: Department of Surveying Engineering, University of New Brunswick. 